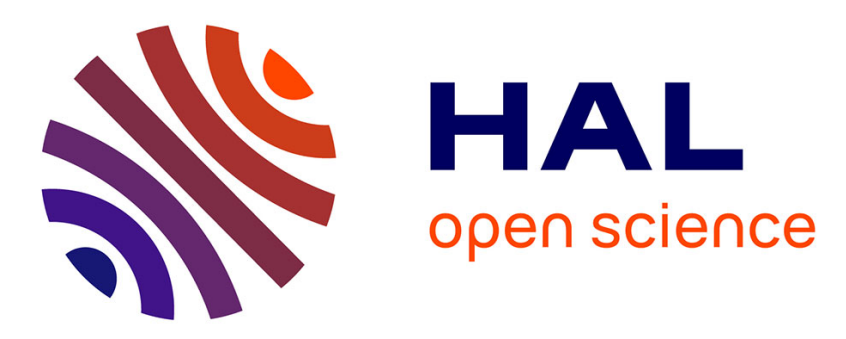

\title{
Révision de quelques pièces humérales plio-pléistocène Sud-Africaines
}

Brigitte Senut

\section{To cite this version:}

Brigitte Senut. Révision de quelques pièces humérales plio-pléistocène Sud-Africaines. Bulletins et Mémoires de la Société d'anthropologie de Paris, 1978, 5 (3), pp.223 - 229. 10.3406/bmsap.1978.1925 . hal-03293648

\section{HAL Id: hal-03293648 \\ https://hal.science/hal-03293648}

Submitted on 21 Jul 2021

HAL is a multi-disciplinary open access archive for the deposit and dissemination of scientific research documents, whether they are published or not. The documents may come from teaching and research institutions in France or abroad, or from public or private research centers.
L'archive ouverte pluridisciplinaire HAL, est destinée au dépôt et à la diffusion de documents scientifiques de niveau recherche, publiés ou non, émanant des établissements d'enseignement et de recherche français ou étrangers, des laboratoires publics ou privés. 


\title{
Révision de quelques pièces humérales plio-pléistocène Sud-
}

\section{Africaines}

\section{Brigitte Sénut}

\section{Résumé}

Résumé. - L'auteur a ré-examiné 5 humérus plio-pléistocènes provenant de Makapansgat (MLD 14, MLD 39, M 2541, M 740) et de Swartkrans (Sk 860). Par comparaison avec des humérus de carnivores et de babouins modernes, il est possible de distinguer parmi ces pièces fragmentaires quelques Cercopithecoidea (MLD 39, M 2541, M 740, Sk 860) et un seulement de ces spécimens fossiles (MLD 14) pourrait être hominidé bien que certains l'attribuent à un babouin ou à un carnivore.

\begin{abstract}
RE-EXAMINATION OF SOME HUMERAL PLIO-PLEISTOCENE PIECES FROM SOUTH-AFRICA Summary. - The author has re-examined 5 plio-pleistocene humérus coming from Makapansgat (MLD 14, MLD 39, M 2541, M 740) and from Swartkrans (Sk 860). By comparison with some modern humerus of carnivores and baboons, it is possible to distinguish among these fragmentary pieces some Cercopithecoidea (MLD 39, M 2541, M 740, Sk 860) and only one of these fossil specimens could be hominid (MLD 14) although some people attributed it to baboon or to a carnivore.
\end{abstract}

\section{Citer ce document / Cite this document :}

Sénut Brigitte. Révision de quelques pièces humérales plio-pléistocène Sud-Africaines. In: Bulletins et Mémoires de la Société d'anthropologie de Paris, XIII ${ }^{\circ}$ Série. Tome 5 fascicule 3, 1978. pp. 223-229;

doi : https://doi.org/10.3406/bmsap.1978.1925

https://www.persee.fr/doc/bmsap_0037-8984_1978_num_5_3_1925

Fichier pdf généré le 09/05/2018 
Bull. et Mém. de la Soc. d'Anthrop. de Paris, t. 5, série XIII, 1978, pp. 223-229

\title{
RÉVISION DE QUELQUES PIËCES HUMÉRALES PLIO-PLEEISTOCËNES SUD-AFRICAINES
}

\author{
par B. Senut (*)
}

\begin{abstract}
RésumÉ. - L'auteur a ré-examiné 5 humérus plio-pléistocènes provenant de Makapansgat (MLD 14, MLD 39, M 2541, M 740) et de Swartkrans (Sk 860). Par comparaison avec des humérus de carnivores et de babouins modernes, il est possible de distinguer parmi ces pièces fragmentaires quelques Cercopithecoidea (MLD 39, M 2541, M 740, Sk 860) et un seulement de ces spécimens fossiles (MLD 14) pourrait être hominidé bien que certains l'attribuent à un babouin ou à un carnivore.
\end{abstract} Swartkrans.

Mots-Clés : Cercopithecoidea, Hominidés, Humérus, Makapansgat, Plio-Pléistocène,
(tkrans.

\section{RE-EXAMINATION OF SOME HUMERAL PLIO-PLEISTOCENE PIECES FROM SOUTH-AFRICA}

SUMMARY. - The author has re-examined 5 plio-pleistocene humerus coming from Makapansgat (MLD 14, MLD 39, M 2541, M 740) and from Swartkrans (Sk 860). By comparison with some modern humerus of carnivores and baboons, it is possible to distinguish among these fragmentary pieces some Cercopithecoidea (MLD 39, M 2541, M 740, Sk 860) and only one of these fossil specimens could be hominid (MLD 14) although some people attributed it to baboon or to a carnivore.

KEY-wORDS : Cer:opithecoidea, Hominids, Humerus, Makapansgat, Plio-Pleistocene, Swartkrans.

Depuis le début du siècle, les sites plio-pléistocènes à Hominidés d'Afrique du Sud ont livré un grand nombre de spécimens dont certains semblent actuellement soulever des problèmes quant à leur attribution. Je me propose ici de revenir sur cinq pièces humérales fossiles que j'ai pu étudier à la Medical School de Johannesburg et au Transvaal Museum de Pretoria lors d'une mission du C.N.R.S. (RCP 292). Il s'agit de quatre fragments osseux provenant de Makapansgat (MLI) 14, MLD 39, M 2541, M 740) et d'un autre découvert à Swartkrans (Sk 860).

(*) Laboratoire d'Anthropologie du Musée de l'Homme. 


\section{I. - MakaPansgat}

Makapansgat comme tous les autres gisements à Australopithèques d'Afrique du Sud se présente comme une grotte creusée dans le calcaire dolomitique précambrien. Les fossiles récoltés dans ce site proviennent de la brèche calcaire qui a fourni plusieurs fragments post-crâniens.

\section{a) MLD 14.}

C'est une portion distale diaphysaire d'humérus droit (fig. 1) découvert en 1949. Il fut décrit par Boné (Boné, 1955) comme appartenant probablement à un Australopithèque, ensuite attribué par Wolpoff (IVolpoff, 1973) à un babouin et puis peut-être à un carnivore par d'autres.

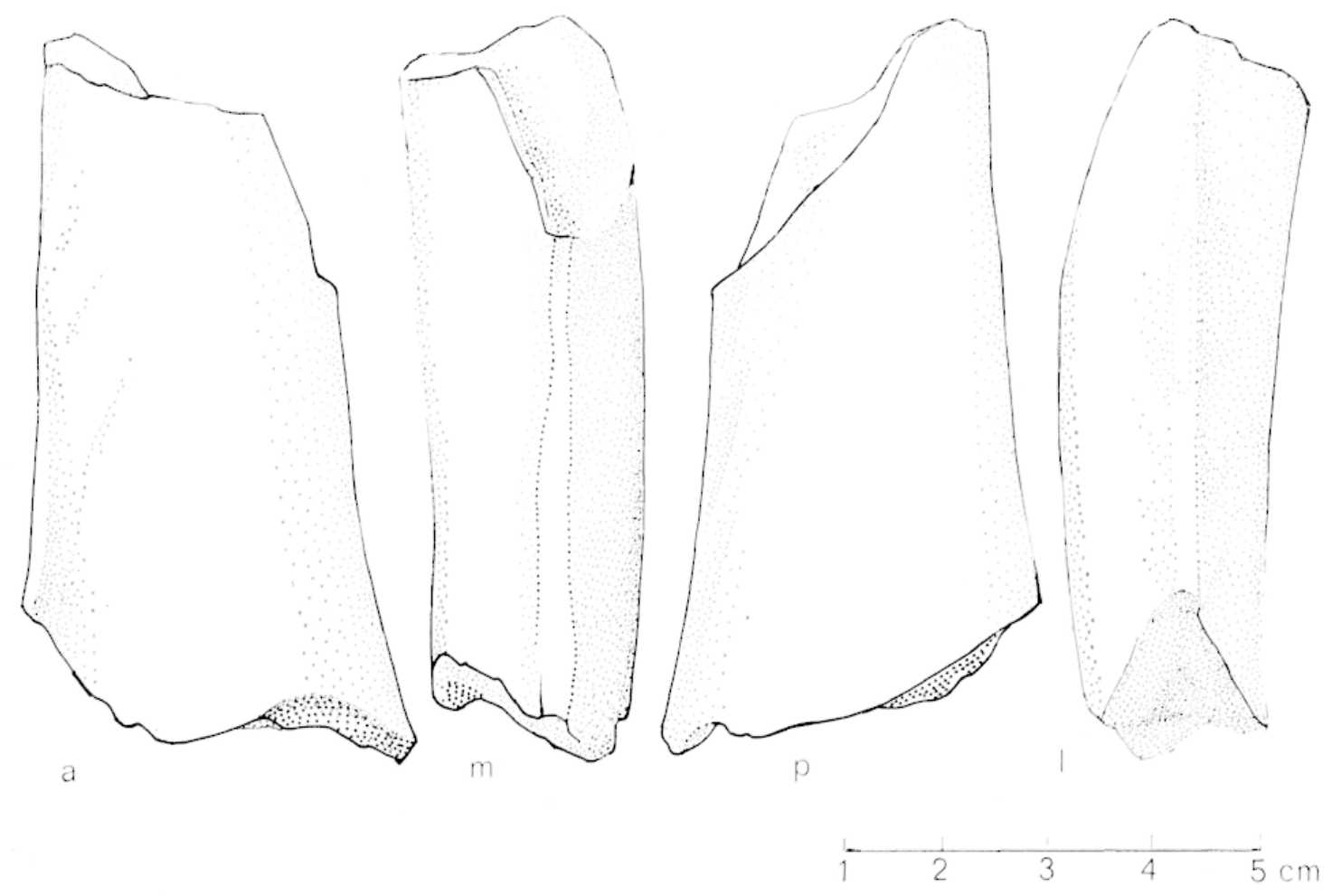

Fig. 1. - MLD 14.

$\mathrm{a}:$ vue antérieure; $\mathrm{m}$ : vue médiale $; \mathrm{p}$ : vue postérieure $; 1$ : vue latérale.

Sur la pièce, aucune surface articulaire n'est visible. L'os a une longueur de $67,5 \mathrm{~mm}$ et présente un fort aplatissement distal (diamètre antéropostérieur $20,7 \mathrm{~mm}$ et diamètre médio-latéral $26,3 \mathrm{~mm}$ dans la région la plus proximale ; diamètre antéro-postérieur $18,6 \mathrm{~mm}$ et diamètre médiolatéral $33,0 \mathrm{~mm}$ dans la partie la plus distale). 
Ses caractères les plus frappants sont : des crètes sus-épitrochléenne et sus-épicondylienne fortes et rectilignes, une face postérieure de l'os bombée, peut-être un léger aplatissement pour l'insertion du musculus brachialis et un fort aplatissement antéro-postérieur du corpus humeri.

Comparé à un certain nombre de carnivores actuels (lion, tigre, ours, hyène, panthère, chien). ce fragment ne peut leur être rapporté. En effet, chez ces animaux, la crête sus-épitrochléenne n'est jamais fortement développée, le corpus humeri présente même un épaississement dans cette région; de plus, celui-ci en vue antérieure est si bombé qu'il semble comprimé médio-latéralement ; d'autre part, la face postérieure de l'os est très aplatie généralement et la crète sus-épicondylienne très forte et très oblique. Pour tous ces caractères très différents de MLD 14 , je ne pense pas que ce dernier puisse être attribué à un carnivore.

Par comparaison avec le babouin, certaines différences se dégagent: ainsi, la face postérieure de l'humérus de Papio est très plate et souvent déprimée antéro-postérieurement, et la crête sus-épitrochléenne n'est pas fortement développée.

Malheureusement, le spécimen MIL) 14 est beaucoup trop fragmentaire pour l'attribuer précisément; mais il faut souligner qu'il ressemble beaucoup à la pièce kényenne KNM ER 739 attribuée à Australopithecus boisei (Leakey, 1971 ; Leakey et al., 1972) en particulier dans le développement des crêtes, dans l'aplatissement du corpus humeri et dans sa taille. On ne peut cependant oublier qu'on connaît à Makapansgat des formes d'Australopithèques graciles de grande taille ('Tobias, 1973).

\section{b) MLD 39.}

Comme les autres spécimens dont il sera question ici, il s'agit d'une extrémité distale d'humérus (fig. 2) rapportée récemment à du babouin mais sans aucune précision (Wolpoff, 1973).

MLI) 39 découvert en 1958 fut décrit par Dart (I)art, 1961) comme étant une spatule façonnée a partir d'un humérus droit d'Australopithèque ; il s'agit en fait d'un humérus gauche ayant perdu toute la région épitrochléenne.

Cette pièce présente tous les caractères d'un Cercopithecoidea moderne ; une trochlea humeri étroite avec une lèvre latérale très faiblement individualisée et une lèvre médiale très projetée vers le bas. On peut également remarquer une fossa olecrani assez profonde et limitée latéralement par une très forte crête ; cette fosse triangulaire est dirigée obliquement avec un apex non pas central mais déporté latéralement. Le capilulum humeri très projeté antérieurement est perpendiculaire à l'axe du corpus humeri. L'epicondylus lateralis très faiblement individualisé se présente sous forme d'une petite bosse latérale située dans le prolongement d'une crête qui semble relativement acérée. 

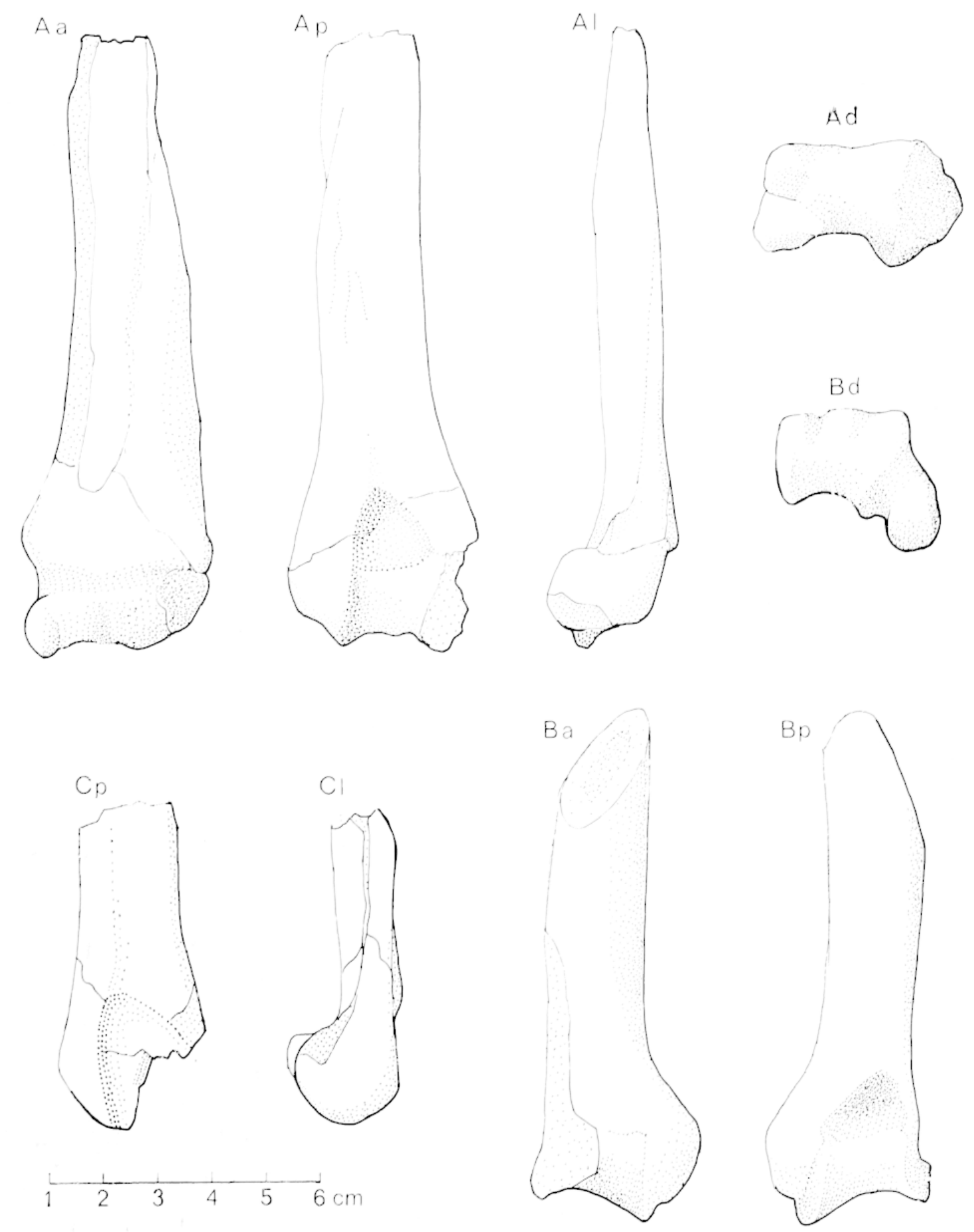

Fig. 2. - A : MLD 39, B : M 2541, C: M 740.

$\mathrm{a}$ : vue antérieure; $\mathrm{p}$ : vue postérieure $; 1$ : vue latérale; $\mathrm{d}$ : vue distale. 
c) M 2541 .

Sur ce spécimen droit (fig. 2), on peut observer une trochlea humeri etroite à lèvre médiale très projetée vers le has, un epicondylus medialis très postérieur et aplati médio-latéralement identique a celui qu'on peut voir chez le babouin moderne, des fossae coronoidea el radialis peu distinctes et une gouttière très netle médialement a la trochlíe en vue postérieure. I)'autre part. la fossa olecrani triangulaire est très profonde.

d) M 740 .

Cette extrémité distale gauche (fig. 2) est très abimce : seul le capilulum humeri est en partie conservé ainsi que les $3 / 4$ de la fossa olecrani.

Ce capilulum présente une certaine projection devant la fossa radialis qui parait large et mal sćparce de la fossa coronoidea. Lepicondylus lateralis, très projeté vers l'avant, montre une face latérale très aplatie.

Ia margo lateralis est nette ; la fossa olecrani, limitée latéralement par une forte crète, est bien triangulaire à apex déporté vers l'extérieur.

Nous nous trouvons donc a Makapansgal en présence dau moins trois Cercopithecoidea et non pas d'Australopithèques. Ie seul spécimen qui peut prèter à réflexion est MLID 14 , mais nous ne pouvons pas ètre catégorique quant a son attribution au genre Australopilhecus, bien qu'il en présente
certains caractères.

\section{II. - Swarthrans}

Sw:artkrans, site dans lequel ont été décrits Paranthropus crassidens et Telanthropus capensis, se présente comme un complexe de trois grottes: "Outer, Inner et Lower Cave ". Celle qui nous intéresse ici tout particulièrement est l'outer Cave qui a livré un certain nombre d'Australopithèques dans une brèche rose où Robinson a mis au jour en 1952 une extrémité distale d'humérus droit ćcrasé en partie. Cet humérus était associé à un fragment de bassin (Sk 50).

\section{Sk 860 .}

Celle pièce est répertorice comme Paranthropus? au Transvaal Museum de Pretoria et comme Paranlhropus crassidens dans Oakley, Camphell et Molleson (Oakley et al., 1977).

Un certain nombre de traits diffèrent de ceux des autres hominidés connus du Plio-Pléislocène et des Hominoidea en général.

Il semble n'y aroir qu'une seule fosse antérieure (les fossae radialis et coronoidea seraient confondues). La lève latérale de la trochlea humeri est très faiblement individualisce et en vue distale se termine au milieu de la surface articulaire au lieu de se prolonger jusqu’au hord latéral de la fossa 
olecrani. La lèvre médiale est bien projetée vers le bas et plus carrée en vue médiale que chez Homo et les autres Hominoidea. La fossa olecrani profonde se présente comme un triangle à sommet déporté latéralement et est limitée par trois murs très raides.

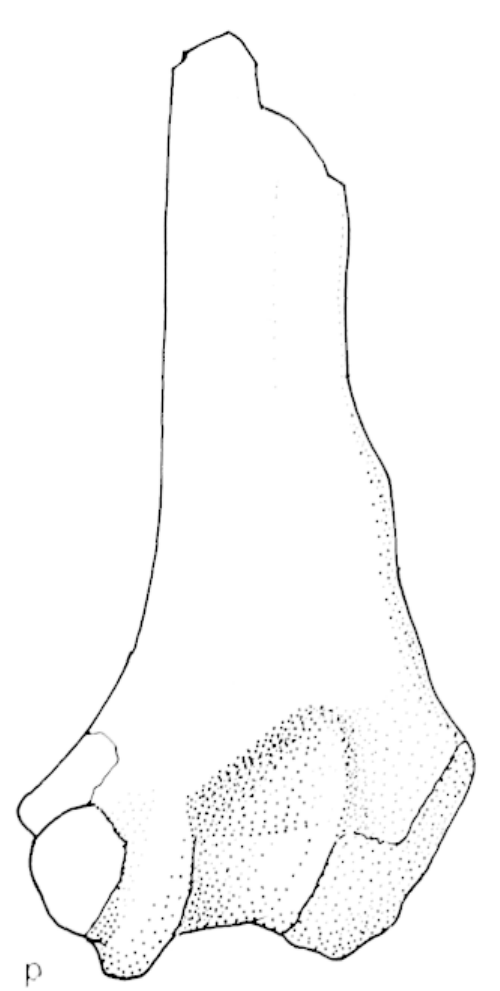

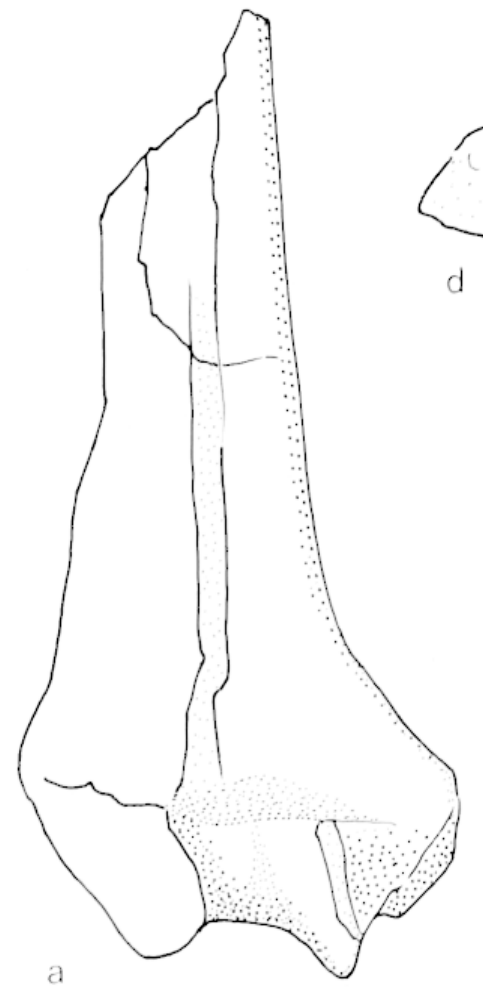

Fig. 3. - Sk 860 .
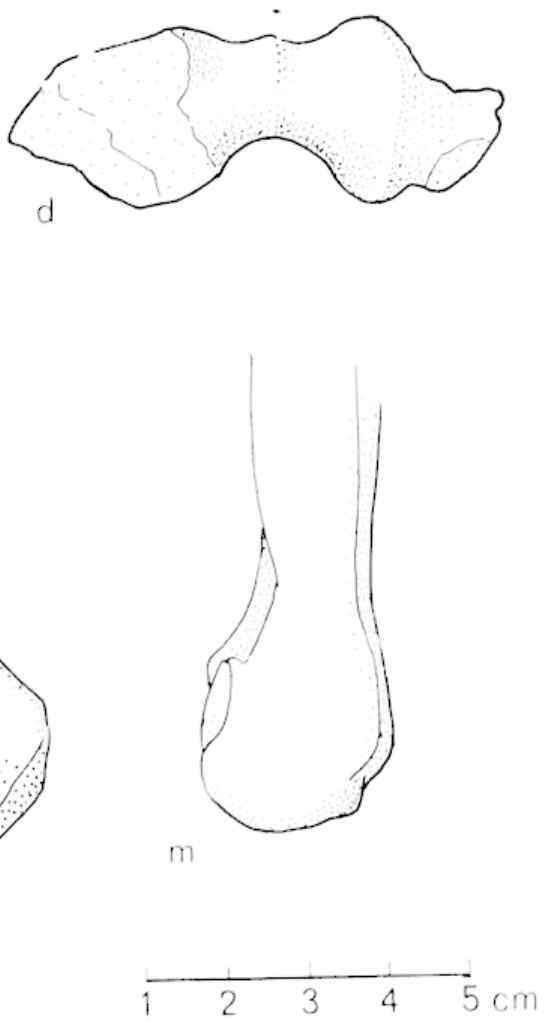

a : vue antérieure $; \mathrm{p}$ : vue postérieure $; \mathrm{m}$ : vue médiale; $\mathrm{d}$ : vue distale.

Ces quelques caractères permettent d'affirmer que nous ne nous trouvons pas en présence d'un Paranthropus, ni d'un Hominoidea, mais bien plus vraisemblablement d'un «babouin ».

Cette pièce est très grosse (largeur biépicondylienne $57,0 \mathrm{~mm}$ ), mais n'oublions pas que l'on connaît à Swartkrans des formes géantes de Cercopithecoidea comme le Dinopithecus par exemple (Brain, 1976).

Ainsi donc, il semble que les confusions avec le babouin, du moins en ce qui concerne le membre supérieur, aient été fréquentes. Je n'ai parlé ici que de l'humérus, mais il faut remarquer que le problème reste également le même avec les pièces radiales.

\section{Remerciements.}

Je dois apporter ici mes vifs remerciements au Professeur P. V. Tobias et au Docteur E. S. Vrba qui m'ont permis d'étudier ces pièces originales, et tout particulièrement à Monsieur Y. Coppens, sans qui ce travail n'aurait pu être réalisé. 


\section{BIBLIOGRAPHIE}

BONÉ (E.), 1955. Quatre fragments post-crâniens du gisement à Australopithèques de Makapansgat (N. Transvaal). L'Anthropologie, t. 59, pp. 462-469.

Brain (C. K.), 1976. A Re-interpretation of the Swartkrans Site and its Remains. S. Afr. J. Sci., V. 72, pp. 141-146.

DarT (R. A.), 1961. An australopithecine scoop made from a right australopithecine upper arm bone. Nature, v. 191, pp. 372-373.

Oakley (K. P.), Campbeli. (B. G.) et Molleson (T. I.), 1977. Catalogue of fossil Hominids. Part I : Africa (Second edition). Trustees of the British Museum (Natural History) London, $1977,223 \mathrm{p}$.

LEAKEY (R. E. F.), 1971. Further evidence of Lower Pleistocene Hominids from East Rudolf, Kenya. Nature, V. 231, pp. 241-245.

Leakey (R. E. F.), Mungai (J. M.) et Walker (A. C.). 1972. New Australopithecines from East Rudolf. Kenya (II). Am. J. Phys. Anthrop., v. 36, no 2, pp. 235-252.

Tobias (P. V.), 1973. New developments in Hominid Paleontology in South and East Africa. Ann. Reviews. Anthrop., V. 2, pp. 311-334.

Wolpoff (M. H.), 1973. Posterior tooth size, body size and diet in South African gracile Australopithecines. Am. J. Phys. Anthrop., v. 39, n ${ }^{0}$ 3, pp. 375-391. 